\title{
Endothelial Cell-Specific Transcriptome Reveals Signature of Chronic Stress Related to Worse Outcome After Mild Transient Brain Ischemia in Mice
}

\author{
Stephanie Wegner $^{1}$ • Ria Uhlemann ${ }^{1}$ • Valérie Boujon ${ }^{1}$ • Burcu Ersoy ${ }^{1}$ • Matthias Endres ${ }^{1,2,3} \cdot$ Golo Kronenberg $^{1,4}$. \\ Karen Gertz ${ }^{1,2}$ (D)
}

Received: 27 May 2019 / Accepted: 23 October 2019 / Published online: 22 November 2019

(C) The Author(s) 2019

\begin{abstract}
Vascular mechanisms underlying the adverse effects that depression and stress-related mental disorders have on stroke outcome are only partially understood. Identifying the transcriptomic signature of chronic stress in endothelium harvested from the ischemic brain is an important step towards elucidating the biological processes involved. Here, we subjected male 129S6/ SvEv mice to a 28-day model of chronic stress. The ischemic lesion was quantified after 30 min filamentous middle cerebral artery occlusion (MCAo) and $48 \mathrm{~h}$ reperfusion by T2-weighted MRI. RNA sequencing was used to profile transcriptomic changes in cerebrovascular endothelial cells (ECs) from the infarct. Mice subjected to the stress procedure displayed reduced weight gain, increased adrenal gland weight, and increased hypothalamic FKBP5 mRNA and protein expression. Chronic stress conferred increased lesion volume upon MCAo. Stress-exposed mice showed a higher number of differentially expressed genes between ECs isolated from the ipsilateral and contralateral hemisphere than control mice. The genes in question are enriched for roles in biological processes closely linked to endothelial proliferation and neoangiogenesis. MicroRNA-34a was associated with nine of the top 10 biological process Gene Ontology terms selectively enriched in ECs from stressed mice. Moreover, expression of mature miR-34a-5p and miR-34a-3p in ischemic brain tissue was positively related to infarct size and negatively related to sirtuin 1 (Sirt1) mRNA transcription. In conclusion, this study represents the first EC-specific transcriptomic analysis of chronic stress in brain ischemia. The stress signature uncovered relates to worse stroke outcome and is directly relevant to endothelial mechanisms in the pathogenesis of stroke.
\end{abstract}

Keywords Psychological stress $\cdot$ Depression $\cdot$ HPA axis $\cdot$ Stroke $\cdot$ Endothelium

Golo Kronenberg and Karen Gertz contributed equally to this work

Electronic supplementary material The online version of this article (https://doi.org/10.1007/s12035-019-01822-3) contains supplementary material, which is available to authorized users.

Karen Gertz

karen.gertz@charite.de

1 Klinik für Neurologie, Charité Campus Mitte,

Charité-Universitätsmedizin Berlin, Charitéplatz 1,

10117 Berlin, Germany

2 DZHK (German Center for Cardiovascular Research), Partner site Berlin, 10115 Berlin, Germany

3 Deutsches Zentrum für Neurodegenerative Erkrankungen (DZNE), 10117 Berlin, Germany

4 University of Leicester and Leicestershire Partnership NHS Trust, Leicester, UK

\section{Introduction}

Chronic psychosocial stress is increasingly recognized as a clinically meaningful cardio- and cerebrovascular risk factor [1-3]. Endothelial dysfunction may represent an important pathophysiological link whereby psychosocial stress confers increased vascular vulnerability $[4,5]$. Even brief episodes of mental stress have been shown to elicit rapid and robust, albeit transient, effects on endothelial function [6,7]. Studies in healthy human subjects indicate that, besides activation of the sympathetic nervous system, dysregulation of the hypothalamic-pituitary-adrenal axis contributes critically to stress-induced endothelial impairment [7-10].

Evidence from experimental animal research indicates that chronic stress increases infarct volume [11, 12]. By combining a mouse model of chronic stress over 4 weeks with a model of mild transient brain ischemia (i.e., 30 min middle cerebral artery occlusion [MCAo]), we previously identified that the 
adverse effects of stress on the ischemic brain involve the endothelium. Mechanistically, both glucocorticoid signaling and a stress-related increase in heart rate were found to cause endothelial dysfunction [13, 14].

Building on this evidence, the current study was undertaken to further characterize the neuroendocrine features of the four week stress model. After confirming that the stress procedure leads to increased lesion volume, we performed RNA sequencing to examine changes in gene expression due to prior stress exposure in ex vivo endothelia harvested from the middle cerebral artery (MCA) territory. We report a transcriptomic signature of chronic stress in MCAo-exposed endothelium and identify upregulation of miRNA-34a as a novel factor promoting ischemic brain injury.

\section{Materials and Methods}

\section{Animals}

All animal studies and experimental procedures were approved by the necessary official committees and conducted in compliance with the requirements set out in the European Communities Council Directive of November 24, 1986 (86/ 609/EEC) and the ARRIVE guidelines [15]. Male 129S6/ SvEv mice raised under specific-pathogen-free (SPF) conditions were provided by the Forschungseinrichtungen für Experimentelle Medizin (FEM) of the Charité Universitätsmedizin Berlin. Young adult male mice $(\sim 9 \pm 1$ weeks old) weighing between 24 and $30 \mathrm{~g}$ were randomly used for experiments. Animals were maintained in a temperature $\left(22{ }^{\circ} \mathrm{C} \pm 2{ }^{\circ} \mathrm{C}\right)$ and humidity $(55 \% \pm 10 \%)$ controlled environment with a 12:12 h light-dark cycle and ad-libitum access to food and water.

\section{Stress Procedure}

A schematic of the experimental setup and timeline is given in Fig. 1a. Briefly, the stress procedure was adapted from previously published protocols $[13,14,16]$.

\section{Exposure to Rat}

At the beginning of the dark phase, a single mouse was situated in a small cage with the following dimensions: height 140 $\mathrm{mm}$, width $167 \mathrm{~mm}$, length $252 \mathrm{~mm}$. This small cage was then placed inside a larger rat cage (height $200 \mathrm{~mm}$, width $375 \mathrm{~mm}$, length $585 \mathrm{~mm}$ ). A rat was introduced into the rat cage for $15 \mathrm{~h}$ (6 p.m. to 9 a.m.). A single session of exposure to rat was also used as the means to induce acute stress.

\section{Restraint Stress}

Animals were placed inside the restraining syringe (internal diameter $30 \mathrm{~mm}$ ) for $1 \mathrm{~h}$ during the dark phase $(7$ p.m. to 8 p.m.).

\section{Tail Suspension Stress}

Mice were suspended by the tail approximately $80 \mathrm{~cm}$ above the ground for $6 \mathrm{~min} /$ day. The procedure started at 7 p.m.

\section{Induction of Cerebral Ischemia}

The standard operating procedure 'Middle cerebral artery occlusion in the mouse' published by Dirnagl and members of the MCAO-SOP group was followed (for a more detailed description of the procedure please refer to $\mathrm{http} / / /$ precedings. nature.com/documents/3492/version/3/files/npre20123492-3. pdf). Briefly, mice were anaesthetized for induction with $1.5 \%$ isoflurane and maintained in $1.0 \%$ isoflurane in $69 \% \mathrm{~N}_{2} \mathrm{O}$ and $30 \% \mathrm{O}_{2}$ using a vaporizer. Left MCAo was induced with an 8 . 0 nylon monofilament coated with a silicone resin/hardener mixture (Xantopren M Mucosa and Activator NF Optosil Xantopren, Heraeus Kulzer GmbH). The filament was introduced into the internal carotid artery up to the anterior cerebral artery. Thereby, the middle cerebral artery and anterior choroidal arteries were occluded. The filament was removed after $30 \mathrm{~min}$ to allow reperfusion.

\section{Magnetic Resonance Imaging}

Successful MCAo was confirmed by magnetic resonance imaging (MRI) using a 7 Tesla rodent scanner (PharmaScan ${ }^{\circledR}$ 70/16, Bruker Corp.) and a 20-mm ${ }^{1} \mathrm{H}-\mathrm{RF}$ quadrature-volume resonator. A T2-weighted 2D turbo spin-echo sequence was used (imaging parameters TR/TE $=4200 / 36 \mathrm{~ms}$, rare factor 8 , 4 averages, 32 axial slices with a slice thickness of $0.5 \mathrm{~mm}$, field of view of $2.56 \times 2.56 \mathrm{~cm}$, matrix size $256 \times 256$ ). Throughout the procedure, animals were anaesthetized with $1-2 \%$ isoflurane in $70 \% \mathrm{~N}_{2} \mathrm{O}$ and $30 \% \mathrm{O}_{2}$. The respiratory rate was monitored with an MRI compatible small animal monitoring and gating system (SA Instruments, Inc.). Lesion volume was evaluated with Analyze 10.0 (AnalyzeDirect, Inc.) and corrected for edema according to a previously published protocol [17].

\section{Measurement of Adrenal Gland Weights and Corticosterone Plasma Levels}

Corticosterone levels in plasma were determined by ELISA according to the manufacturer's instructions (RE52211, IBL International $\mathrm{GmbH}$ ). After sacrifice, the adrenal glands were dissected and weighed. 
a

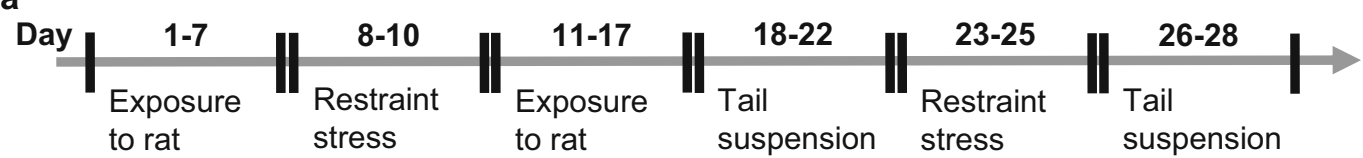

b

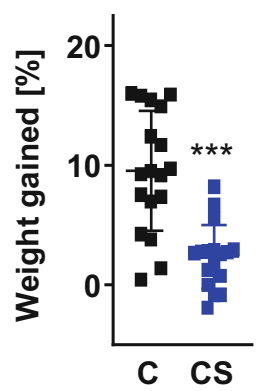

C

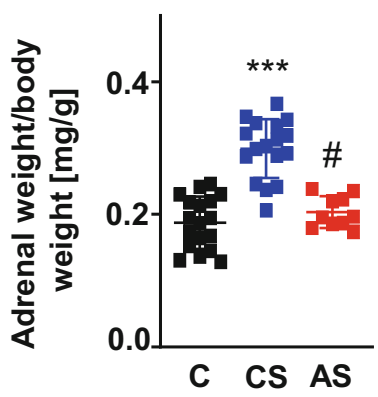

d

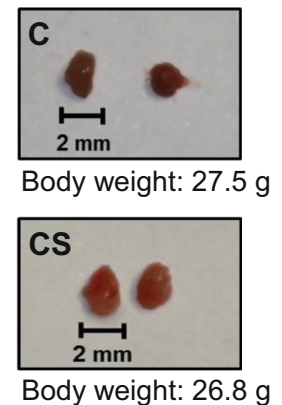

e

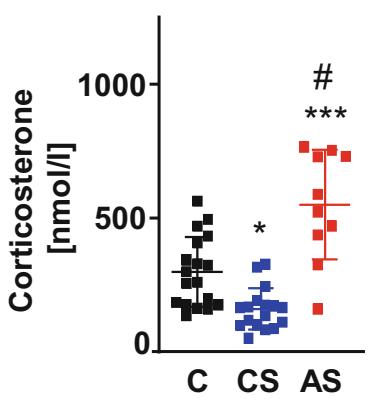

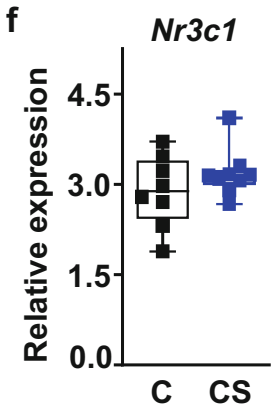
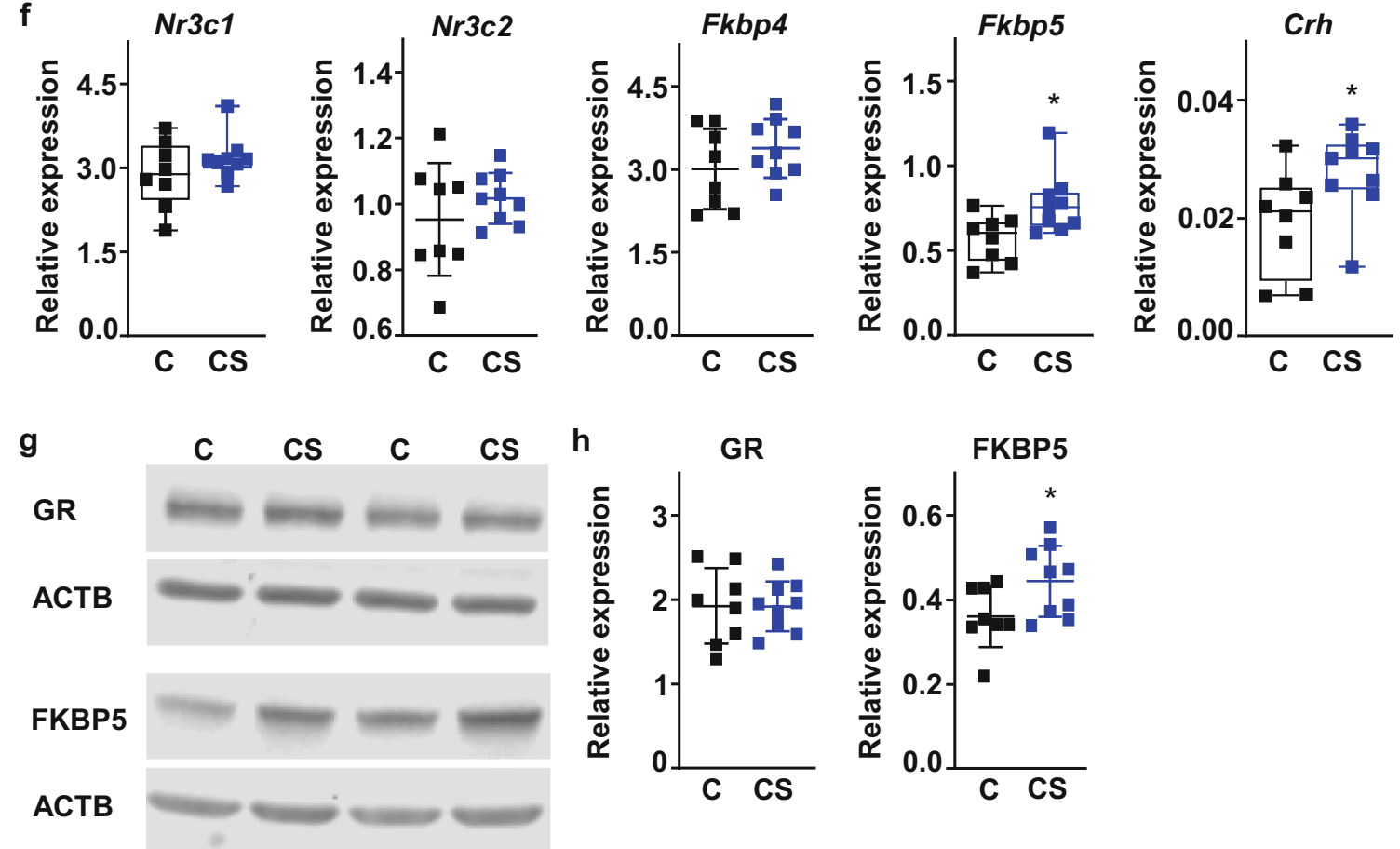

Fig. 1 Characterization of the neuroendocrine effects of the chronic stress paradigm. a Schematic diagram of experimental design. b Body weight was recorded before the beginning and at the end of the chronic stress procedure. Chronically stressed mice gain less body weight than unstressed control animals. C: $n=18$, CS: $n=17$. Unpaired $t$ test. $t=$ 5.262, $* * * p<0.001$. c, d Chronic stress increases the weight of the adrenal glands. C: $n=18$, CS: $n=17$, AS: $n=10$. One-way ANOVA $\mathrm{F}(2,42)=40.14, p<0.001$ with Tukey's multiple comparison test: $* * * p<$ $0.001 \mathrm{CS}$ versus $\mathrm{C}, \# p<0.001 \mathrm{CS}$ versus AS. e Corticosterone plasma levels were measured at the beginning of the light cycle. C: $n=18$, CS: $n$ $=17$, AS: $n=10$. One-way ANOVA F $(2,42)=26.20, p<0.001$ with Tukey's multiple comparison test: $* p<0.05$ CS versus $\mathrm{C}$, $* * * p<0.001$ AS versus $\mathrm{C}, \# p<0.001 \mathrm{CS}$ versus AS. f Hypothalamic mRNA transcription of genes associated with corticosteroid signaling. $\mathrm{Nr} 3 \mathrm{cl}$, nuclear

\section{RNA Isolation}

Total RNA was extracted from hypothalamus and from ex vivo endothelial cells (ECs) using the NucleoSpin ${ }^{\circledR}$ receptor subfamily 3 group $\mathrm{C}$ member 1 . $N r 3 c 2$, nuclear receptor subfamily 3 group C member 2. Fkbp4, FK506 binding protein 4. Fkbp5, FK506 binding protein 5. Crh, corticotropin releasing hormone. Relative mRNA expression is reported as the value normalized to tripeptidyl peptidase 2 (Tpp2) for each sample. C: $n=8$, CS: $n=9$. Mann-Whitney $U$ test. Nr3c1: $U=27, p=0.423$. Fkbp5: $U=12, * p<0.05$. Crh: $U=14, * p$ $<0.05$. Unpaired $t$ test. Nr3c2: $t=1.016, p=0.326$. Fkbp4: $t=1.214, p=$ 0.244. g Representative Western blots of GR and FKBP5 protein expression in hypothalamic homogenates. GR, glucocorticoid receptor. FKBP5, FK506 binding protein 51. ACTB, $\beta$-actin. h Densitometric analysis. Values were normalized to $\beta$-actin. C: $n=8$, CS: $n=9$. Unpaired $t$ test. GR: $t=0.029, p=0.977$. FKBP5: $t=2.173,{ }^{*} p<0.05$. C, unstressed control mice. CS, chronically stressed mice. AS, acutely stressed mice

XS Kit according to the manufacturer's protocol (MACHEREY-NAGEL GmbH \& Co. KG). RNA isolation from ECs was performed without the addition of carrier RNA. 


\section{Quantitative Polymerase Chain Reactions}

Synthesis of cDNA was performed with 300 U M-MLV reverse transcriptase (Promega Corp.), $20 \mathrm{U}$ RNasin ${ }^{\circledR}$ ribonuclease inhibitor (Promega Corp.), 4.76 mM DTT (Promega Corp.), $1.43 \mathrm{mM}$ PCR nucleotide mix (Promega Corp.), and $6.28 \mu \mathrm{M}$ random primers (Roche Diagnostics $\mathrm{GmbH}$ ). For PCR amplification, we used gene-specific primers (Table 1) and LightCycler® 480 SYBR Green I Master (Roche Diagnostics $\mathrm{GmbH})$. Polymerase chain reaction conditions were as follows: preincubation $95^{\circ} \mathrm{C}, 10 \mathrm{~min} ; 95^{\circ} \mathrm{C}, 10 \mathrm{~s}$, primer-specific annealing temperature, $10 \mathrm{~s}, 72{ }^{\circ} \mathrm{C}, 15 \mathrm{~s}(45$ cycles). Crossing points of amplified products were determined using the Second Derivative Maximum Method (LightCycler® 480 Version 1.5.0 SP4, Roche). Quantification of messenger RNA expression was relative to tripeptidyl peptidase (Tpp) 2 [18]. The specificity of polymerase chain reaction products was evaluated by melting curve analysis and electrophoresis on a 1.5\% agarose gel.

\section{MiRNA Isolation and Quantification}

Total RNA (including the microRNA fraction) was isolated from ischemic brain tissue using the Direct-zol ${ }^{\mathrm{TM}}$ RNA MiniPrep Kit (Zymo Research Corp.). Tissue was homogenized with TRIzol ${ }^{\mathrm{TM}}$ Reagent (Invitrogen ${ }^{\mathrm{TM}}$ ) and TissueLyser LT (Qiagen) for $3 \times 5 \mathrm{~min}$ at $50 \mathrm{~Hz}$. Transcription of $500 \mathrm{ng}$ total RNA into cDNA and real-time PCR was carried out with the miScript PCR Starter Kit (Qiagen). Custom miScript Primer Assays were used to detect miR-34a-5p, miR-34a-3p, and RNU6B (Qiagen). The level of miRNA expression in ischemic brain tissue of control mice and of chronically stressed mice was normalized to RNU6B (RNU6-2) using the $2^{(-\Delta \Delta \mathrm{Ct})}$ method [19]. Melting curve analysis and agarose gel electrophoresis confirmed specificity of polymerase chain reaction products.

\section{Western Blot}

After sacrifice, brains were quickly removed, flash frozen in dry ice-cooled isopentane, and stored until further use. The hypothalamus was dissected on a cold plate $\left(-20^{\circ} \mathrm{C}\right)$ according to Franklin and Paxinos (1997). Protein concentration was determined with the Pierce ${ }^{\mathrm{TM}}$ BCA Protein Assay Kit (ThermoFisher Scientific Inc.). Equal amounts of protein were loaded on $10 \%$ SDS-polyacrylamide gels and blotted onto Immobilon®-FL PVDF membranes (Merck KGaA). Nearinfrared fluorescent signals were detected with the Odyssey® CLx Infrared Imaging System (LI-COR, Inc.). Densitometric quantification of band intensity was performed with the Image Studio ${ }^{\text {TM }}$ Lite Software (LI-COR, Inc.). The following primary antibodies were used: rabbit antiglucocorticoid receptor (\#12041, Cell Signaling Technology,
Inc.) 1:1000; rabbit anti-FKBP5 (\#711292, ThermoFisher Scientific Inc.) 1:1000; mouse anti-beta actin (\#ab8226, Abcam plc.) 1:2000. Secondary antibodies were used as follows: donkey anti-rabbit 800 (\#925-32213, LI-COR, Inc.) 1:15000; donkey anti-mouse 680 (\#925-32213, LI-COR, Inc.) 1:15000.

\section{Ex vivo Isolation of Brain ECs}

Control (C) and chronically stressed mice (CS) were perfused transcardially with $0.9 \%$ saline. Brains were quickly removed. The ipsilateral ischemic MCA territory, as well as the corresponding area in the contralateral hemisphere, was carefully dissected and placed in cold PBS $\left(4^{\circ} \mathrm{C}\right)$. Brain tissue of 3-5 animals was pooled. $\mathrm{CD} 31+$ cells were enriched from brain tissue using a MACS protocol combined with prior myelin removal (Miltenyi Biotec $\mathrm{GmbH}$ ). To ensure a high purity of ECs, an additional FACS step was performed. Cells were stained with DAPI and antibodies against CD31, CD146, and CD45. Single cells that were CD45-/DAPI-/CD31+/ CD146+ were separated from the remainder of the suspension using a BD FACSAria ${ }^{\mathrm{TM}}$ II flow cytometer. For FACS staining, the following antibodies were used: anti-mouse CD31Alexa Fluor® 488 (\#102514, BioLegend, Inc.); anti-mouse CD45-APC (\#130-102-544, Miltenyi Biotec GmbH); antimouse CD146 (LSEC)-PE (\#130-102-319, Miltenyi Biotec $\mathrm{GmbH})$.

\section{RNA Sequencing of Ex vivo ECs}

Assessment of RNA quality, cDNA synthesis, library preparation, sequencing, data pre-processing, alignment, and principal component analysis (PCA) was conducted by LGC Genomics GmbH (Berlin, Germany). Libraries were prepared with the Encore Rapid DR Multiplex system (NuGEN Technologies, Inc.) according to the manufacturer's directions and sequenced with $150 \mathrm{bp}$ paired-end reads on the Illumina ${ }^{\circledR}$ NextSeq ${ }^{\mathrm{TM}}$ 500. Data pre-processing involved the following steps: demultiplexing of all libraries with the Illumina ${ }^{\circledR}$ bcl2fastq 2.17.1.14 software, clipping of sequencing adapter remnants from all raw reads, merging of forward and reverse reads using BBMerge 34.48, SMRT concatamer adapter detection and read splitting, filtering of poly-A reads, and filtering of rRNA sequences with riboPicker 0.4.3 [20]. RNA-seq data have been deposited at the Gene Expression Omnibus (GEO) [21] under GEO accession number GSE122345.

\section{Analysis of Differentially Expressed Genes}

A total of 400 million reads were aligned to the reference genome (Mus musculus; GRCm38; Ensembl version 84) using STAR 2.4.1b [22]. Reads mapping to rRNA or tRNA regions were filtered post-alignment. Counting of STAR- 
Table 1 Oligonucleotide sequences used in quantitative real-time polymerase chain reactions

\begin{tabular}{lll}
\hline Gene & Sense & Antisense \\
\hline Crh & CTA CCA AGG GAG GAG AAG AGA G & CTG CTC CGG CTG CAA GAA ATT C \\
Fkbp4 & CGT GCT CAA GGT CAT CAA GAG AG & GTT GCC ACA GCA ATA TCC CAA GC \\
$F k b p 5$ & GCA GGG TGA AGA TAT CAC TAC G & CCA ATG TCC CAG GCT TTG ATA AC \\
$N r 3 c 1$ & GAC GTG TGG AAG CTG TAA AGT C & CCA GGT TCA TTC CAG CTT GAA G \\
$N r 3 c 2$ & CAC ACG GTG ACC TGT CAT CTA G & CAT AGT GAC ACC CAG AAG CCT C \\
$T p p 2$ & CTT CTA TCC AAA GGC TCT CAA GG & CTC TCC AGG TCT CAC CAT CAT G \\
Sirt 1 & CCA GAC CCT CAA GCC ATG TTT G & CTG CAA CCT GCT CCA AGG TAT C \\
\hline
\end{tabular}

aligned reads was conducted using htseq-count [23]. FPKM values (fragments per kilobase per million fragments mapped) and differentially expressed genes (DEGs) analyses were calculated with Cuffdiff 2.1.1 by LGC Genomics GmbH (Berlin, Germany). The raw $p$ values from the statistical tests were adjusted for multiple testing by the Benjamini-Hochberg false discovery rate (FDR) method. Genes with an FDR-adjusted $p$ value $<0.05$ and fold change $\geq 2$ or $\leq-2$ were considered to be DEGs. When a gene cannot be detected in samples derived from one side of the brain, the $\log 2$ (fold change) yields a value of infinity. In those instances in which the $\log 2$ (fold change) yielded infinity and the FDR-adjusted $p$ value was $<0.05$, the gene in question was still not regarded as differentially expressed because these genes also showed a very low mean count of $<4$ on the other side of the brain ( 5 genes in the control mice [Gm13755, Gm3191, Igkv4-58, Gm6064, 1700017I07Rik]; 2 genes in the chronically stressed animals [Gm24447, Gm11686]). The Venn diagram (Fig. 3c) was created with a web-based tool [24]. The expression levels of different marker genes were visualized with heatmapper [25]. Specific markers for each cell population were collated from different publications [26-33].

\section{Gene Ontology Analysis}

Gene ontology (GO) enrichment analysis was performed with $\mathrm{g}$ :Profiler [34; Ensembl version 91]. The size of functional categories was limited to between 5 and 5000 genes so as to reduce redundancy in $\mathrm{GO}$ annotation. The BenjaminiHochberg false discovery rate (FDR) correction was calculated to adjust for multiple testing. GO annotations with an adjusted $p$ value $<0.01$ were considered to be significantly enriched. Finally, REVIGO was used to filter out redundant categories [35].

\section{Statistics}

Except for the stress experiments, all procedures and analyses were performed in a blinded fashion. Results are presented as individual values and as mean $\pm \mathrm{SD}$ or as boxplot with median and interquartile range as appropriate. Mice were excluded from this study either when MRI yielded evidence of intracerebral hemorrhage $(\mathrm{C}: 0 \%, \mathrm{CS}: 3 \%)$ or failed to confirm successful MCAo (C: 19\%, CS: 21\%). Statistical analysis was performed using GraphPad Prism version 7 or 8 (GraphPad Software, Inc.). Normality testing was done using D'Agostino-Pearson omnibus test ( $n \geq 8)$ or Shapiro-Wilk test $(n<8)$. Since the present study was designed as an exploratory investigation, we did not correct for multiple comparisons.

\section{Results}

\section{Neuroendocrine Effects of the Chronic Stress Paradigm}

A schematic diagram of the experimental design is given in Fig. 1a. Briefly, three different stressors (i.e., exposure to rat, restraint stress, and tail suspension stress) were applied in an alternating fashion over the course of the experiment. Chronically stressed mice (CS) gained significantly less weight than control animals (C), which were left unperturbed in their home cages (Fig. 1b). Adrenal weight was significantly increased in CS mice after completion of the stress paradigm (Fig. 1c, d). Acute stress, i.e., a single session of exposure to rat before sacrifice early on the following morning, resulted in significantly increased circulating corticosterone levels and no relevant change in adrenal gland weight relative to $\mathrm{C}$ mice (Fig. 1c, e). By contrast, CS mice showed significantly reduced corticosterone plasma concentrations relative to $\mathrm{C}$ mice at the end of the experiment (Fig. 1e). Next, we studied mRNA transcription of genes associated with corticosteroid signaling in the left hypothalamus (Fig. 1f). Expression of glucocorticoid receptor $(\mathrm{Nr} 3 \mathrm{cl}) \mathrm{mRNA}$ and expression of mineralocorticoid receptor $(\mathrm{Nr} 3 \mathrm{c} 2) \mathrm{mRNA}$ did not differ significantly between $\mathrm{C}$ and CS mice. However, CS mice showed significantly increased mRNA transcription of $F k b p 5$ and $C r h$ (Fig. 1f). Further, Western blot analysis of homogenates prepared from right hypothalamus confirmed increased FKBP5 protein expression in CS mice (Fig. 1g, h). 


\section{Chronic Stress Impacts the Brain's Sensitivity to Ischemic Injury}

Next, we combined the chronic stress procedure with a model of mild transient brain ischemia (Fig. 2a). C and CS mice were used. Two days after 30 min MCAo/reperfusion, lesion size was measured in vivo using T2-weighted MRI. Lesion volume was found to be significantly increased in CS mice compared to $\mathrm{C}$ mice (Fig. 2b). Endothelial mechanisms have been implicated in linking psychological stress with stroke vulnerability $[13,14]$. We therefore performed a gene expression analysis using ex vivo ECs harvested separately from the ischemic MCA territory and the corresponding area in the contralateral non-ischemic hemisphere (Fig. 2c). C and CS samples were pooled from groups of 3-5 mice (Fig. 2c). As illustrated in Fig. 2d, cells pre-enriched for CD31 were FACS purified to obtain CD45-/DAPI-/CD31+/ CD146+ brain endothelia. An analysis of cell typespecific genes confirmed the purity of the FAC-sorted cells (Fig. 2e). Finally, a principal component analysis (PCA) performed on the RNA-seq data yielded two factors (Fig. 2f).

\section{A Transcriptomic Signature of Chronic Stress in MCAo-Exposed Endothelium}

Volcano plots show genes that were increased or decreased by more than two-fold $(p<0.05)$ in ipsilateral compared to contralateral endothelium (Fig. 3a, b). The number of differentially expressed genes (DEGs) was higher in endothelia from CS mice than in endothelia from $\mathrm{C}$ mice. Figure $3 \mathrm{c}$ summarizes the number of DEGs in C and CS mice as well as the intersection of genes regulated similarly in both experimental groups. A complete list of genes included in each category is provided in supplementary material ESM 2-4. Additionally, Fig. 3d summarizes the number of DEGs in different log2 (fold change) categories. RNA-seq did not yield differences in gene expression between ECs harvested from the contralateral hemisphere of $\mathrm{C}$ mice and ECs harvested from the contralateral hemisphere of CS mice.

Next, we performed a GO term analysis for the DEGs in each group. The annotation rates of the DEGs were $84.9 \%$ and 96.3\% in ECs from C and CS mice, respectively. ESM 1a summarizes biological process GO terms that were simultaneously enriched in ECs from both C and CS mice $(p<0.01)$. Additional biological process GO terms emerged in CS mice. The top 10 of these terms are given in Fig. 3e. The biological processes in question are highly relevant to endothelial viability and function and highlight the effects of prior stress priming on the transcriptomic response to MCAo. The complete lists of GO terms enriched exclusively in $\mathrm{C}$ or $\mathrm{CS}$ mice are given in ESM $1 \mathrm{~b}$ and $1 \mathrm{c}$.
Fig. 2 Chronic stress increases early lesion size after MCAo. a Experimental setup. Animals were either subjected to the 28-day stress procedure (CS) or were left unperturbed (C). On day 29, C and CS mice underwent 30 min MCAo/reperfusion. Successful MCAo was confirmed at $48 \mathrm{~h}$ by T2-weighted MRI. Mice were sacrificed $72 \mathrm{~h}$ after MCAo. b Chronic stress resulted in increased acute lesion sizes after $30 \mathrm{~min}$ MCAo. C: $n=25$, CS: $n=26$. Mann-Whitney $U$ test. $U=213$, $* p<0.05$. c, d Endothelial cells (ECs) were harvested from the ipsilateral (ipsi; i.e., infarcted MCA territory) and contralateral (contra; i.e., corresponding area on the non-infarcted side) hemispheres of C and CS mice. Cells from 3-5 mice were pooled for each sample. d Gating strategy for flow cytometric sorting of MACS-purified CD31+ cells. e The heatmap confirms strong expression of endothelial marker genes in all samples. $\mathbf{f}$ Principal component analysis (PCA) plot of all RNA-seq data. The twodimensional scatter plot represents the differential patterns of gene expression in ECs harvested from $\mathrm{C}$ and CS mice after $30 \mathrm{~min}$ $\mathrm{MCAo} /$ reperfusion. FPKM, fragments per kilobase per million fragments mapped

\section{Upregulation of MicroRNA-34a Promotes Ischemic Injury}

MicroRNA-34a is increased by atheroprone oscillatory shear stress and promotes endothelial senescence [36, 37]. MiR-34a has previously been shown to repress SIRT1 expression [37, 38]. MiR-34a-5p and miR-34a-3p are the mature sequences of miR-34a.

MiR-34a was one of the transcripts found to be only differentially regulated in ECs from CS mice (Fig. 3b). Crucially, miR-34a was associated with nine of the top 10 biological process GO terms exclusively enriched in CS endothelium (Fig. 3e). We therefore investigated the relationship between expression of mature miR-34a-5p and miR-34a-3p in ischemic brain tissue and MRI lesion size.

There was considerable variance in infarct size among mice in the CS group, possibly reflecting partial resilience to the adverse effects of chronic stress in a subgroup of CS mice. For this reason, we divided CS mice into two groups based on their infarct sizes: (1) 'CS large' group with an infarct size above the median of the CS group, (2) 'CS small' group. Expression of miR-34a-5p and miR34a-3p was significantly increased while Sirt 1 mRNA expression was significantly decreased in the 'CS large' group relative to both the ' $\mathrm{CS}$ small' group and $\mathrm{C}$ mice (Fig. $4 \mathrm{a}-\mathrm{c}$ ). Interestingly, mRNA transcription of Fkbp 5 observed from ischemic whole brain tissue did not differ significantly between experimental groups (Fig. 4d). However, we still found a moderate positive relationship between mRNA transcription of Fkbp5 in ischemic brain tissue and infarct volume (Fig. 4e).

Expression of miR-34a-5p and miR-34a-3p was positively related to infarct size (Fig. 4f, g) and negatively related to Sirt 1 mRNA expression (Fig. 4h, i). Finally, transcription of Sirt1 mRNA was negatively related to the size of the ischemic lesion (Fig. 4j). 


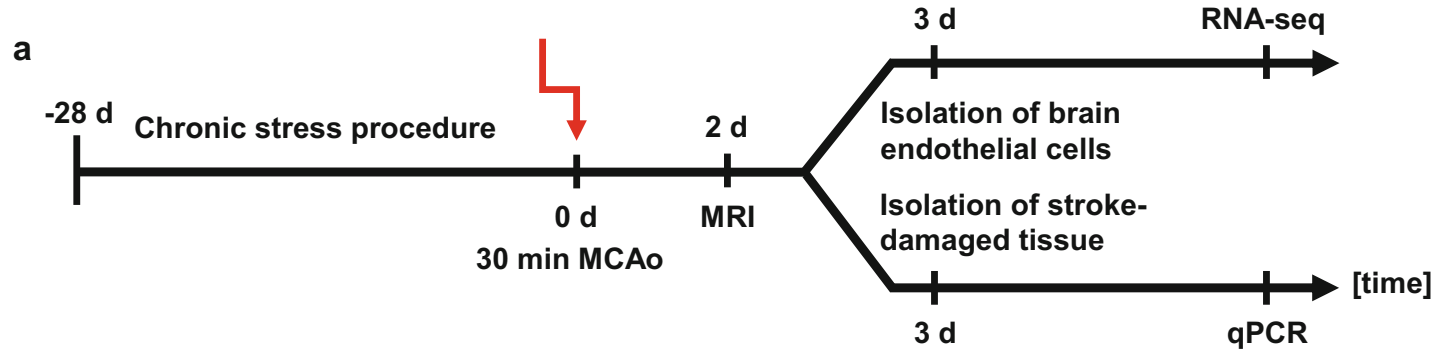

b
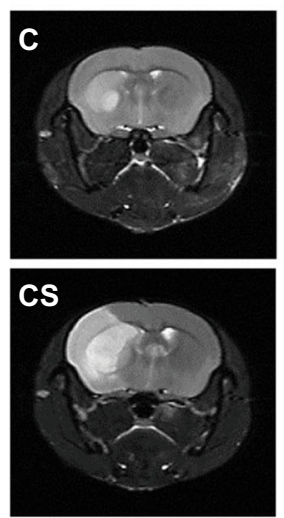

d
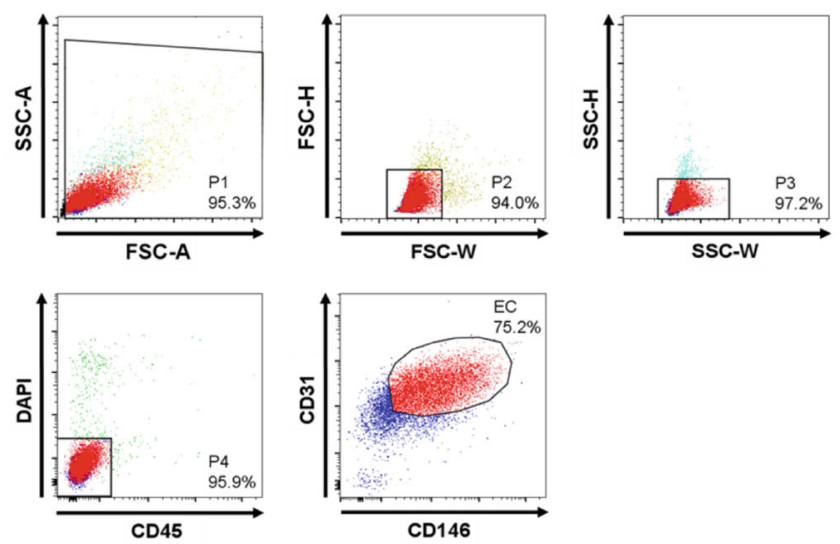

f

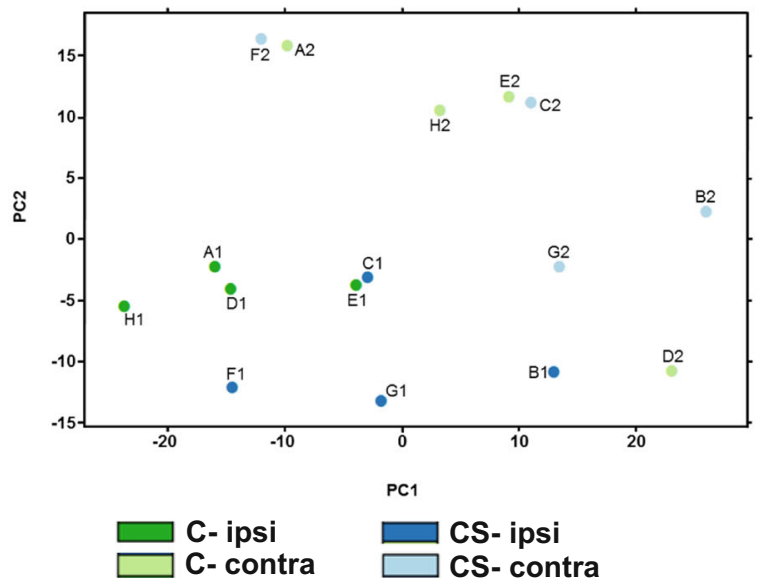

C

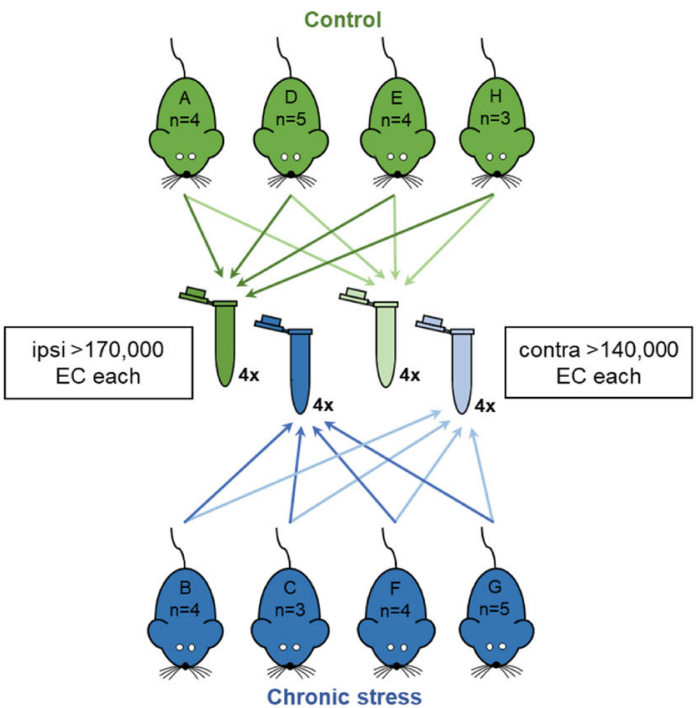

e C CS

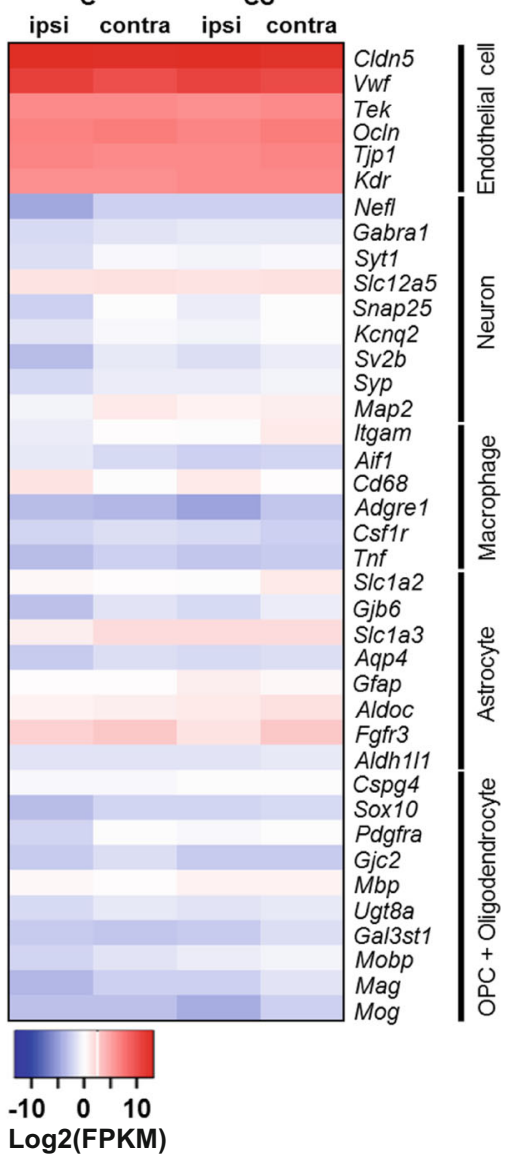




\section{Discussion}

While this study focuses chiefly on endothelial mechanisms, it is noted that the adverse effects of chronic stress are farreaching and clearly not restricted to a single cell type in the brain. For example, the expression of neurotrophins in brain tissue is strongly impacted by stress and corticosteroids, which, in turn, may affect cellular plasticity and neuronal vulnerability to ischemia $[39,40]$. With this being acknowledged, however, one key advantage to our reductionist approach is that it provides a new and hitherto barely considered angle on the complex pathways linking stress and stroke.

This study yielded the following key findings: (1) The chronic stress paradigm exerted robust effects at multiple levels, including, among others, reduced weight gain, increased adrenal gland weight, and increased hypothalamic FKBP5 mRNA and protein expression. (2) Using T2weighted MR imaging, we replicate our earlier finding that the chronic stress paradigm sensitizes experimental animals to the effects of $30 \mathrm{~min}$ MCAo/reperfusion. (3) Next, we used RNA sequencing to profile gene expression changes in endothelia harvested from the ischemic brain as an effect of prior stress. We identified a distinct transcriptomic signature characterized by a higher number of DEGs in MCAo-exposed ECs derived from CS mice. Biological process GO terms enriched in ECs from CS mice are highly relevant to cell viability and function and include terms such as "cell proliferation," "negative regulation of biological process," "positive regulation of apoptotic process," "cell death," "negative regulation of signal transduction," and "negative regulation of cell proliferation." MicroRNA-34a is associated with nine of the top 10 biological process GO terms enriched in CS endothelium. (4) Expression of mature miR-34a-5p and miR-34a-3p in ischemic brain tissue was positively related to infarct size measured by MRI and negatively related to Sirt1 mRNA expression.

Both reduced body weight $[16,41,42]$ and increased body weight [43-45] have been observed with different rodent stress models. Factors that may explain differences between studies include the age, sex, and strain of the experimental animals, the kind of stressor used, and, importantly, the duration of the stressor [45, 46]. The chronic stress model was initially developed by Strekalova et al. with a view to producing a depression-like syndrome in male C57BL/6 mice [16]. For stroke experiments, our group adapted this model to 129S6/SvEv mice [13, 14], which, because of the nature of their cerebrovascular anatomy, lend themselves particularly well to transient intraluminal MCAo [47]. Our finding of reduced body weight in CS mice relative to non-stressed controls is in accord with the initial report by Strekalova et al. [16]. In line with our earlier study [13], the present investigation also confirms dysregulation of the HPA axis in the form of increased adrenal glands in CS mice. Furthermore, we show here that, in our model, chronic stress leads to
Fig. 3 Transcriptomic analysis of brain ECs. ECs were harvested from C and CS mice $72 \mathrm{~h}$ after 30 min MCAo. Upregulation or downregulation of gene expression is relative to gene expression in endothelia harvested from the contralateral hemisphere. a, b Volcano plots of all genes quantified by RNA-seq. The negative $\log 10$ of the $p$ value is plotted on the $y$ axis. The $x$ axis is the $\log 2$ of the fold change between endothelial gene expression in the ipsilateral and contralateral sides. a Gene expression in control (C) animals. b Gene expression in chronically stressed mice (CS). Green dots represent genes which are significantly upregulated or downregulated in $\mathrm{C}$ mice. Blue dots represent genes which are significantly upregulated or downregulated in CS mice. Yellow dots represent genes significantly regulated in both $\mathrm{C}$ and CS mice. Gray dots represent genes without significant regulation. The two vertical columns of pink dots in the chart are genes for which no expression was detected in ECs harvested from either the contralateral or the ipsilateral hemisphere, so $\log 2$ (fold change) values were arbitrarily set to \pm 20 (instead of \pm infinity). Note that genes with a $\log 2$ (fold change) value of infinity and FDR $<0.05$ were not considered as differentially expressed due to low mean read counts. $\mathbf{c}$ Venn diagram showing the number and overlap of differentially regulated genes in ECs from C and CS mice. Green circle: endothelial gene expression in $\mathrm{C}$ mice; blue circle: endothelial gene expression in CS mice. $\mathbf{d}$ The table summarizes the number of differentially expressed genes (DEGs) in different log2(fold change) categories. e The top 10 biological process GO terms that were only detected in CS mice (see supplementary data for entire list)

upregulation of both Crh mRNA and FKBP5 mRNA and protein in hypothalamus. FKBP5 mRNA and protein expression is induced upon glucocorticoid receptor stimulation, providing an intracellular negative feedback loop for glucocorticoid receptor activity [48]. Together, these results strongly point to depression-like HPA axis hyperactivity and glucocorticoid resistance in mice subjected to 4 weeks of chronic stress $[49,50]$. Interestingly, measurements in morning plasma performed at the end of the stress regime revealed decreased corticosterone concentrations in CS mice. A similar result has previously been reported in a chronic social defeat paradigm [45]. As plasma was taken 1 day after the end of the stress procedure and the animals had been left undisturbed in their home cages during the preceding dark phase, reduced trough corticosterone levels in CS mice most likely reflect adaptive changes of the HPA axis to chronic stress [45].

In the current study, we used the MCAo model to apply a cell-specific transcriptomic approach to uncover stress signatures in cerebrovascular endothelium. In doing so, we focused on molecular and physiological endpoints along with early lesion size on MRI. To our knowledge, this is the first report investigating the effects of chronic psychological stress on the endothelial transcriptome. Expression profiling of cerebrovascular endothelium following MCAo has likewise not yet been reported in the literature.

After completion of the 4-week chronic stress procedure, experimental mice were subjected to $30 \mathrm{~min}$ MCAo/reperfusion and sacrificed after an interval of $72 \mathrm{~h}$. Our results, therefore, reflect the enduring effects of prior stress on stroke outcome. We acknowledge that our study does not address the connection between 


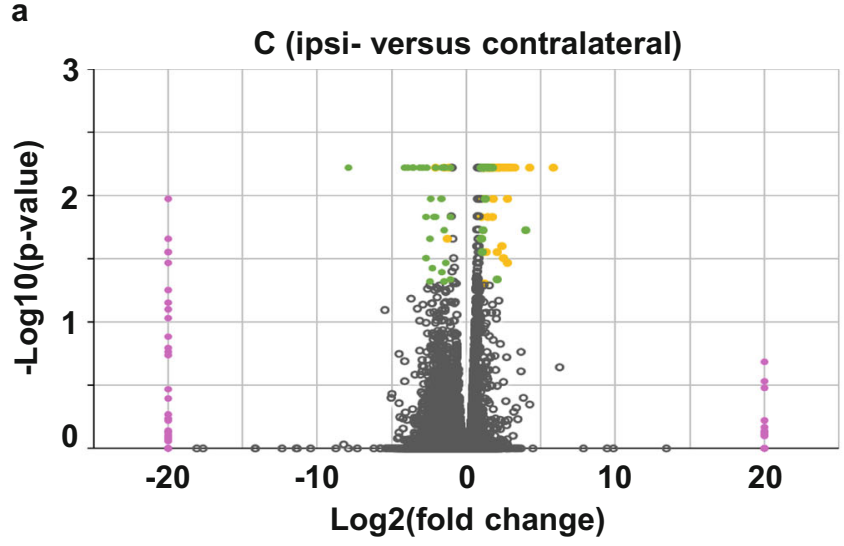

b

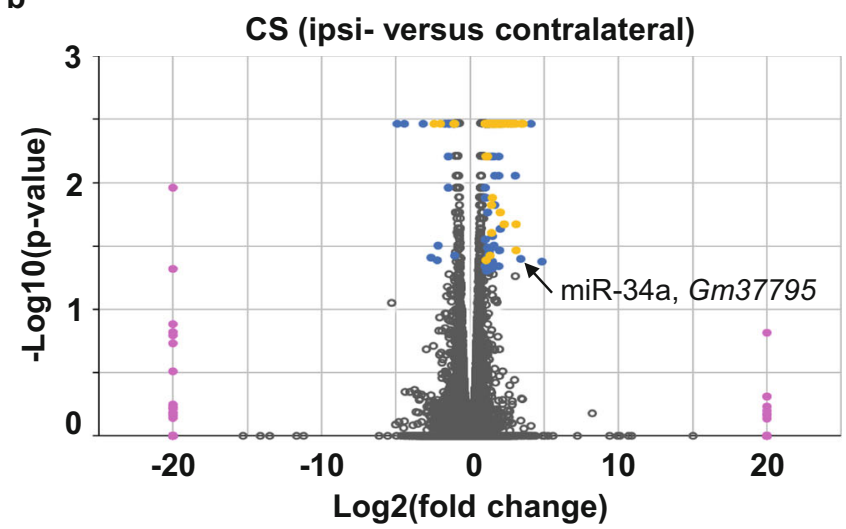

- Significantly regulated in C - Significantly regulated in CS Significantly regulated in C and CS o Nonsignificantly regulated

- Infinity log2(fold change) values

C

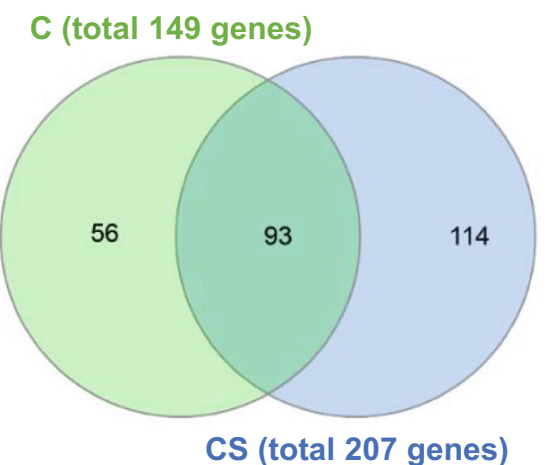

e

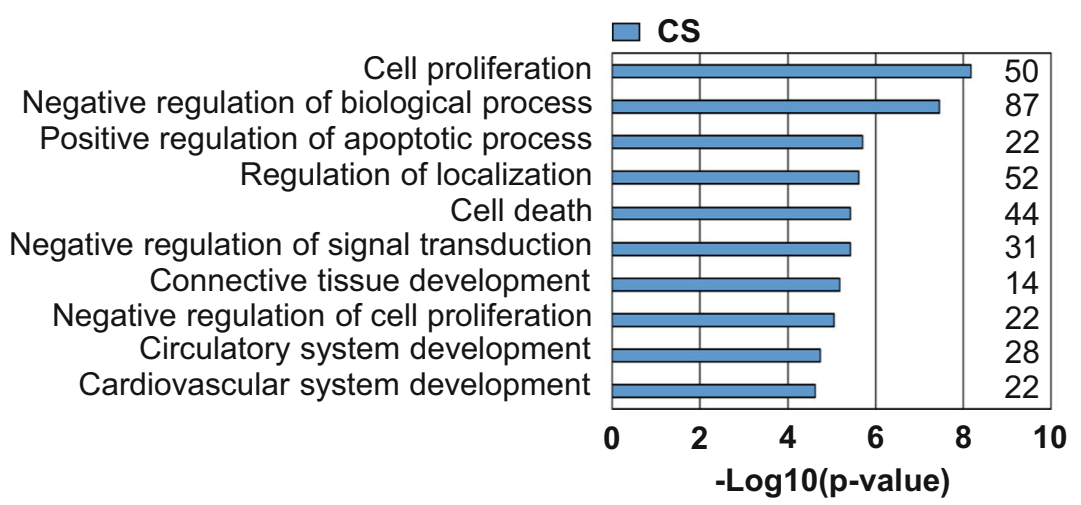

chronic stress, depressive-like behaviors, and functional outcomes after transient brain ischemia. However, in agreement with earlier studies using histological evaluation of infarct size [12-14, 51], T2-weighted imaging at $48 \mathrm{~h}$ confirmed a stress-induced increase in lesion volume.

ECs were isolated from the ipsilateral and contralateral MCA territory using sequential rounds of MACS and FACS. First, ECs were enriched by CD31 magnetic beads. Then, progressive FACS gating was used to remove cellular aggregates and cellular debris, as well as CD31 expressing hematopoietic cells [52, 53]. Purity of ECs was corroborated by robust expression of endothelial cell-specific marker genes alongside the absence of marker genes for other brain cells. Interestingly, we did not detect significant differences in gene expression 

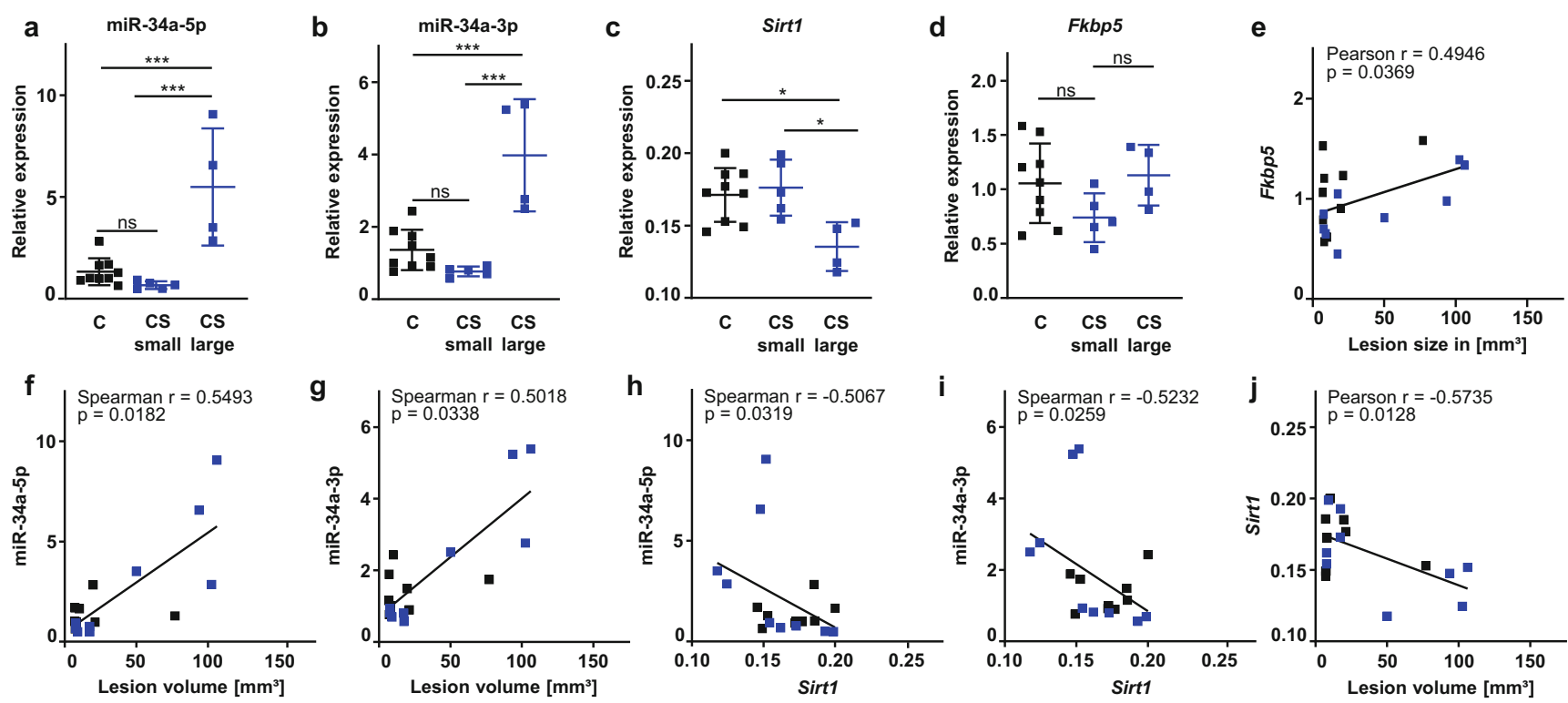

c $\square \mathrm{cs}$

Fig. 4 Expression of miR-34a microRNAs is positively related to lesion size and inversely related to Sirt 1 mRNA expression in ischemic whole brain. Mice were killed $72 \mathrm{~h}$ after 30 min MCAo/reperfusion. As described in the main text, CS mice were divided into two groups based on their infarct sizes - 'CS large' group and 'CS small' group. Expression levels of miR-34a-5p, miR-34a-3p, Sirt1, and Fkbp5 were assessed in ischemic whole brain tissue. MiR-34a values are expressed as fold change relative to RNU6B and one control mouse using the $2^{(-\Delta \Delta \mathrm{Ct})}$ method. Relative Sirt 1 and $F k b p 5$ mRNA expression values were calculated using the $\Delta \mathrm{CT}$ method corrected for primer efficiency and normalized to Tpp2

between ECs harvested from the contralateral hemispheres of $\mathrm{C}$ and CS mice. This most likely suggests that, in our experimental setup, the 3-day interval between stress and sacrifice was too long for the effects of as housekeeping gene. a-d C: $n=9$, 'CS small' group: $n=5$, 'CS large' group: $n=4$. $\mathbf{e}-\mathbf{j} N=9$ mice per group. a One-way ANOVA $\mathrm{F}(2,15)=$ $16.28, p<0.001$ with Tukey's multiple comparison test: $* * * p<0.001$. b One-way ANOVA $\mathrm{F}(2,15)=19.82, p<0.001$ with Tukey's multiple comparison test: $* * * p<0.001$. c One-way ANOVA $\mathrm{F}(2,15)=6.575, p$ $<0.01$ with Tukey's multiple comparison test: $* p<0.05$. d One-way ANOVA F $(2,15)=2.138, p=0.152$. $\mathbf{e}-\mathbf{j}$ Pearson's correlation coefficient or Spearman's rank correlations were computed depending on whether data were normally distributed or not

psychological stress to persist on the transcriptomic level in otherwise unperturbed brain endothelium. Of course, the effects of the intervening MCAo also have to be taken into consideration as the procedure itself is

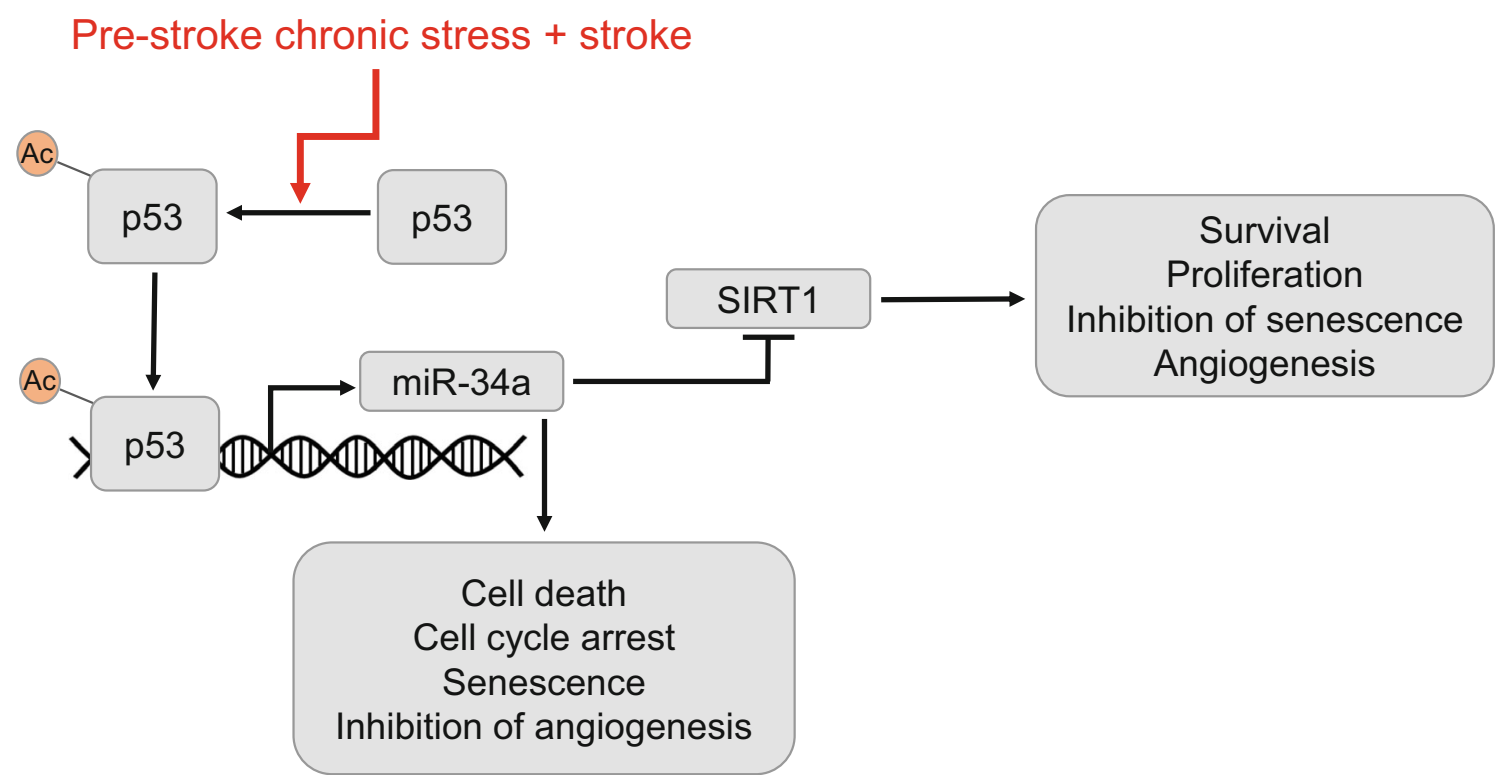

Fig. 5 Upregulation of miR-34a in brain endothelia of CS mice after MCAo. The combination of ischemic injury and chronic stress results in increased transcriptional activity of p53, most likely through a posttranslational modification such as increased acetylation [38]. In consequence, miR-34a is upregulated, resulting in repression of SIRT1 and modulation of proliferation and apoptosis $[37,38]$ 
a strong stressor and leads to elevations in circulating corticosterone [e.g., 54].

In this analysis, we focused on genes that were either increased or decreased by more than two-fold $(p<0.05)$ in ipsilateral compared to contralateral ECs. As can be seen from the Volcano plots in Fig. 3, relatively few transcripts fell into this category. Biological process GO terms enriched in both $\mathrm{C}$ and CS mice are summarized in ESM 1a and reflect the broad cellular effects of ischemia on ECs. As described above, more DEGs were detected in CS than in C mice. Further exploration of our genomic findings will prove useful in elucidating the adverse effects of stress on ECs in the pathogenesis of stroke. For example, the upregulation of Angpt2 in CS mice may point to altered blood vessel growth in the ischemic brain as angiopoietin-2 has been shown to impair revascularization after limb ischemia [55]. Adamts9, which was similarly upregulated in ipsilateral CS endothelium, has recently been identified as an endogenous angiogenesis inhibitor that operates cell-autonomously in ECs [56]. By contrast, mRNA expression of the brain-specific angiogenesis inhibitor 1 (Adgrb1) was not altered in CS endothelium, but strongly downregulated in ipsilateral ECs from $\mathrm{C}$ mice [57]. In the context of these observations, it is important to note that endothelial cell proliferation and angiogenesis are crucial regenerative mechanisms post-stroke [e.g., 58-61].

Finally, microRNA-34a was selectively increased in CS endothelium from the ischemic brain. This finding is highly significant in the context of a confluence of research pointing to the importance of vascular mechanisms and regenerative angiogenesis for stroke outcome [58, 62, 63]. We speculate that pre-stroke stress in tandem with stroke injury enhances transcriptional activity of p53 most likely through its acetylation [38]. In turn, acetylated p53 upregulates miR-34a, thereby repressing SIRT1 and modulating proliferation and apoptosis $[37,38]$. A schema of the putative underlying miR-34a pathway in brain endothelia is presented in Fig. 5 .

There is ample evidence in the literature to indicate that changes in miR-34a and miR-34a target genes such as Sirt1 will directly impact ischemic injury and, hence, infarct size. MiR-34a has been identified as a key driver of cardiovascular senescence [37, 64]. Moreover, anti-miR34a treatment following experimental myocardial infarction (MI) via coronary artery ligation significantly improved cardiac function post-MI [65]. Expression of miR-34a is elevated in senescent human umbilical cord vein endothelial cells as well as in heart and spleen of older mice [37]. MiR-34a induces G1 arrest, thereby blocking endothelial cell proliferation [37]. Conversely, inhibition of miR-34a by antisense oligonucleotides has been shown to increase capillary density in a mouse model of myocardial infarction, highlighting the favorable effects of inhibition of miR-34a on cardiac endothelium in the ischemic border zone [64]. Finally, SIRT1 is expressed by vascular endothelium in brain and has been shown to mediate cerebrovascular protection by facilitating NO-dependent vascular relaxation in a murine model of cerebral hypoperfusion induced by bilateral common carotid artery stenosis [66]. A growing body of evidence suggests that miRNAs may be released into the bloodstream or cerebrospinal fluid (CSF), making them attractive candidates for biomarker development. It is, therefore, especially notable that exciting new research has reported increased miR-34a-5p concentrations in blood and CSF of patients suffering from major depression [67]. Due to limited EC numbers, 3 to 5 samples per experimental condition had to be pooled in the current study. For this reason, it was not possible to directly correlate endothelial miR-34a expression to infarct size. However, in a subsequent experiment, we examined the relationship between MRI lesion size and expression of mature miR-34a-5p and miR-34a$3 p$ in ischemic whole brain tissue. SIRT1 expression has been shown to be repressed by miR-34a [38]. Our analysis shows that expression of miR-34a-5p and miR-34a-3p was positively related to infarct size whereas Sirtl transcription was negatively related to infarct size. An obvious caveat to this finding is that miR-34a may be expressed by neurons as well as by other non-neuronal cells besides endothelia [68, 69].

In conclusion, this study identifies a transcriptomic signature of chronic stress in endothelia harvested from the ischemic murine brain. This stress signature relates to worse stroke outcome and is directly relevant to endothelial mechanisms in the pathogenesis of stroke.

Acknowledgments The technical assistance of Bettina Herrmann, Melanie Kroh, Renate Franke, and Stefanie Balz is gratefully acknowledged. We also thank the Charité Core Facility '7T Experimental MRIs' and the Deutsches Rheuma-Forschungszentrum Berlin 'Flow Cytometry Core Facility' (FCCF) for excellent support.

Funding Information This work was supported by the Deutsche Forschungsgemeinschaft (Exc257 to M.E.; KR 2956/4-1 und KR 2956/ 6-1 to G.K.; GE 2576/3-1 und GE 2576/5-1 to K.G.), the Bundesministerium für Bildung und Forschung (CSB to M.E., K.G. and G.K.), the German Center for Neurodegenerative Diseases (DZNE to M.E.), the German Center for Cardiovascular Research (DZHK to M.E. and K.G.), and the Corona Foundation (to M.E.).

\section{Compliance with Ethical Standards}

Conflict of Interest The authors declare that they have no competing interests.

Open Access This article is distributed under the terms of the Creative Commons Attribution 4.0 International License (http:// creativecommons.org/licenses/by/4.0/), which permits unrestricted use, distribution, and reproduction in any medium, provided you give appropriate credit to the original author(s) and the source, provide a link to the Creative Commons license, and indicate if changes were made.

\section{References}

1. Rosengren A, Hawken S, Ounpuu S, Sliwa K, Zubaid M, Almahmeed WA, Blackett KN, Sitthi-amorn C et al (2004) Association of psychosocial risk factors with risk of acute 
myocardial infarction in 11119 cases and 13648 controls from 52 countries (the INTERHEART study): case-control study. Lancet. 364:953-962

2. O'Donnell MJ, Xavier D, Liu L, Zhang H, Chin SL, Rao-Melacini $P$ et al (2010) Risk factors for ischaemic and intracerebral haemorrhagic stroke in 22 countries (the INTERSTROKE study): A case-control study. Lancet. 376:112-123

3. Everson-Rose SA, Roetker NS, Lutsey PL, Kershaw KN, Longstreth WT, Sacco RL et al (2014) Chronic stress, depressive symptoms, anger, hostility, and risk of stroke and transient ischemic attack in the multi-ethnic study of atherosclerosis. Stroke. 45:23182323

4. Kershaw KN, Lane-Cordova AD, Carnethon MR, Tindle HA, Liu K (2017) Chronic stress and endothelial dysfunction: the multiethnic study of atherosclerosis (MESA). Am J Hypertens 30:75-80

5. Mausbach BT, Roepke SK, Ziegler MG, Milic M, von Känel R, Dimsdale JE et al (2010) Association between chronic caregiving stress and impaired endothelial function in the elderly. J Am Coll Cardiol 55:2599-2606

6. Ghiadoni L, Donald AE, Cropley M, Mullen MJ, Oakley G, Taylor M, O'Connor G, Betteridge J et al (2000) Mental stress induces transient endothelial dysfunction in humans. Circulation. 102: 2473-2478

7. Spieker LE, Hürlimann D, Ruschitzka F, Corti R, Enseleit F, Shaw S, Hayoz D, Deanfield JE et al (2002) Mental stress induces prolonged endothelial dysfunction via endothelin-A receptors. Circulation. 105:2817-2820

8. Broadley AJM, Korszun A, Abdelaal E, Moskvina V, Jones CJH, Nash GB, Ray C, Deanfield J et al (2005) Inhibition of cortisol production with metyrapone prevents mental stress-induced endothelial dysfunction and baroreflex impairment. J Am Coll Cardiol 46:344-350

9. Eriksson M, Johansson K, Sarabi M, Lind L (2007) Mental stress impairs endothelial vasodilatory function by a beta-adrenergic mechanism. Endothelium. 14:151-156

10. Hijmering ML, Stroes ESG, Olijhoek J, Hutten BA, Blankestijn PJ, Rabelink TJ (2002) Sympathetic activation markedly reduces endothelium-dependent, flow-mediated vasodilation. J Am Coll Cardiol 39:683-688

11. DeVries AC, Joh H-D, Bernard O, Hattori K, Hurn PD, Traystman RJ, Alkayed NJ (2001) Social stress exacerbates stroke outcome by suppressing Bcl-2 expression. Proc Natl Acad Sci U S A 98:1182411828

12. Sugo N, Hurn PD, Morahan MB, Hattori K, Traystman RJ, DeVries AC (2002) Social stress exacerbates focal cerebral ischemia in mice. Stroke. 33:1660-1664

13. Balkaya M, Prinz V, Custodis F, Gertz K, Kronenberg G, Kroeber J, Fink K, Plehm R et al (2011) Stress worsens endothelial function and ischemic stroke via glucocorticoids. Stroke. 42:3258-3264

14. Custodis F, Gertz K, Balkaya M, Prinz V, Mathar I, Stamm C, Kronenberg G, Kazakov A et al (2011) Heart rate contributes to the vascular effects of chronic mental stress: effects on endothelial function and ischemic brain injury in mice. Stroke. 42:1742-1749

15. Kilkenny C, Browne WJ, Cuthill IC, Emerson M, Altman DG (2010) Improving bioscience research reporting: the ARRIVE guidelines for reporting animal research. PLoS Biol 8:e1000412

16. Strekalova T, Spanagel R, Bartsch D, Henn FA, Gass P (2004) Stress-induced anhedonia in mice is associated with deficits in forced swimming and exploration. Neuropsychopharmacology. 29:2007-2017

17. Gerriets T, Stolz E, Walberer M, Müller C, Kluge A, Bachmann A et al (2004) Noninvasive quantification of brain edema and the space-occupying effect in rat stroke models using magnetic resonance imaging. Stroke. 35:566-571

18. Nishida Y, Sugahara-Kobayashi M, Takahashi Y, Nagata T, Ishikawa K, Asai S (2006) Screening for control genes in mouse hippocampus after transient forebrain ischemia using high-density oligonucleotide array. J Pharmacol Sci 101:52-57

19. Livak KJ, Schmittgen TD (2001) Analysis of relative gene expression data using real-time quantitative PCR and the 2(-Delta Delta C(T)) Method. Methods. 25:402-408

20. Schmieder R, Lim YW, Edwards R (2012) Identification and removal of ribosomal RNA sequences from metatranscriptomes. Bioinformatics. 28:433-435

21. Edgar R, Domrachev M, Lash AE (2002) Gene Expression Omnibus: NCBI gene expression and hybridization array data repository. Nucleic Acids Res 30:207-210

22. Dobin A, Davis CA, Schlesinger F, Drenkow J, Zaleski C, Jha S, Batut P, Chaisson M et al (2013) STAR: ultrafast universal RNAseq aligner. Bioinformatics. 29:15-21

23. Anders S, Pyl PT, Huber W (2015) HTSeq - a Python framework to work with high-throughput sequencing data. Bioinformatics. 31: 166-169

24. Heberle H, Vaz Meirelles G, da Silva FR, Telles GP, Minghim R (2015) InteractiVenn: a web-based tool for the analysis of sets through Venn diagrams. BMC Bioinformatics 16:169

25. Babicki S, Arndt D, Marcu A, Liang Y, Grant JR, Maciejewski A et al (2016) Heatmapper: web-enabled heat mapping for all. Nucleic Acids Res 44(Web Server):W147-W153

26. Cahoy JD, Emery B, Kaushal A, Foo LC, Zamanian JL, Christopherson KS et al (2008) A transcriptome database for astrocytes, neurons, and oligodendrocytes: a new resource for understanding brain development and function. J Neurosci 28:264-278

27. Wiedenmann B, Franke WW (1985) Identification and localization of synaptophysin, an integral membrane glycoprotein of Mr 38,000 characteristic of presynaptic vesicles. Cell. 41:1017-1028

28. Dinsmore JH, Solomon F (1991) Inhibition of MAP2 expression affects both morphological and cell division phenotypes of neuronal differentiation. Cell. 64:817-826

29. Chawla A, Barak Y, Nagy L, Liao D, Tontonoz P, Evans RM (2001) PPAR- $\gamma$ dependent and independent effects on macrophage-gene expression in lipid metabolism and inflammation. Nat Med 7:48-52

30. Ito D, Imai Y, Ohsawa K, Nakajima K, Fukuuchi Y, Kohsaka S (1998) Microglia-specific localisation of a novel calcium binding protein, Iba1. Brain Res Mol Brain Res 57:1-9

31. Zakrzewska A, Cui C, Stockhammer OW, Benard EL, Spaink HP, Meijer AH (2010) Macrophage-specific gene functions in Spi1directed innate immunity. Blood. 116:e1-e11

32. Mustonen T, Alitalo K (1995) Endothelial receptor tyrosine kinases involved in angiogenesis. J Cell Biol 129:895-898

33. Stevenson BR, Siliciano JD, Mooseker MS, Goodenough DA (1986) Identification of ZO-1: a high molecular weight polypeptide associated with tight junction (zonula occludens) in a variety of epithelia. J Cell Biol 103:755-766

34. Reimand J, Arak T, Adler P, Kolberg L, Reisberg S, Peterson H et al (2016) g:Profiler-a web server for functional interpretation of gene lists (2016 update). Nucleic Acids Res 44(Web Server):W83-W89

35. Supek F, Bošnjak M, Škunca N, Šmuc T (2011) REVIGO summarizes and visualizes long lists of gene ontology terms. PLoS One 6: e21800

36. Fan W, Fang R, Wu X, Liu J, Feng M, Dai G, Chen G, Wu G (2015) Shear-sensitive microRNA-34a modulates flow-dependent regulation of endothelial inflammation. J Cell Sci 128:70-80

37. Ito T, Yagi S, Yamakuchi M (2010) MicroRNA-34a regulation of endothelial senescence. Biochem Biophys Res Commun 398:735740

38. Yamakuchi M, Ferlito M, Lowenstein CJ (2008) miR-34a repression of SIRT1 regulates apoptosis. Proc Natl Acad Sci U S A 105: 13421-13426

39. Smith MA, Makino S, Kvetnansky R, Post RM (1995) Stress and glucocorticoids affect the expression of brain-derived neurotrophic 
factor and neurotrophin-3 mRNAs in the hippocampus. J Neurosci 15:1768-1777

40. Endres M, Fan G, Hirt L, Fujii M, Matsushita K, Liu X, Jaenisch R, Moskowitz MA (2000) Ischemic brain damage in mice after selectively modifying BDNF or NT4 gene expression. J Cereb Blood Flow Metab 20:139-144

41. Magariños AM, McEwen BS (1995) Stress-induced atrophy of apical dendrites of hippocampal CA3c neurons: involvement of glucocorticoid secretion and excitatory amino acid receptors. Neuroscience. 69:89-98

42. Warren BL, Vialou VF, Iñiguez SD, Alcantara LF, Wright KN, Feng J, Kennedy PJ, Laplant Q et al (2013) Neurobiological sequelae of witnessing stressful events in adult mice. Biol Psychiatry 73: $7-14$

43. Hammamieh R, Chakraborty N, De Lima TCM, Meyerhoff J, Gautam A, Muhie S et al (2012) Murine model of repeated exposures to conspecific trained aggressors simulates features of posttraumatic stress disorder. Behav Brain Res 235:55-66

44. Patterson ZR, Khazall R, MacKay H, Anisman H, Abizaid A (2013) Central ghrelin signaling mediates the metabolic response of C57BL/6 male mice to chronic social defeat stress. Endocrinology. 154:1080-1091

45. Savignac HM, Finger BC, Pizzo RC, O’Leary OF, Dinan TG, Cryan JF (2011) Increased sensitivity to the effects of chronic social defeat stress in an innately anxious mouse strain. Neuroscience. 192:524-536

46. Sutanto W, de Kloet ER (1994) The use of various animal models in the study of stress and stress-related phenomena. Lab Anim 28: 293-306

47. Maeda K, Hata R, Hossmann K-A (1998) Differences in the cerebrovascular anatomy of C57Black/6 and SV129 mice. Neuroreport. 9:1317-1319

48. Binder EB (2009) The role of FKBP5, a co-chaperone of the glucocorticoid receptor in the pathogenesis and therapy of affective and anxiety disorders. Psychoneuroendocrinology. 34(Suppl 1): S186-S195

49. Westberry JM, Sadosky PW, Hubler TR, Gross KL, Scammell JG (2006) Glucocorticoid resistance in squirrel monkeys results from a combination of a transcriptionally incompetent glucocorticoid receptor and overexpression of the glucocorticoid receptor cochaperone FKBP51. J Steroid Biochem Mol Biol 100:34-41

50. Pariante CM, Miller AH (2001) Glucocorticoid receptors in major depression: relevance to pathophysiology and treatment. Biol Psychiatry 49:391-404

51. Madrigal JLM, Caso JR, de Cristóbal J, Cárdenas A, Leza JC, Lizasoain I, Lorenzo P, Moro MA (2003) Effect of subacute and chronic immobilisation stress on the outcome of permanent focal cerebral ischaemia in rats. Brain Res 979:137-145

52. Kim S-J, Kim J-S, Papadopoulos J, Wook Kim S, Maya M, Zhang F, He J, Fan D et al (2009) Circulating monocytes expressing CD31: implications for acute and chronic angiogenesis. Am J Pathol 174:1972-1980

53. Wilkinson R, Lyons BA, Roberts D, Wong M-X, Bartley PA, Jackson DE (2002) Platelet endothelial cell adhesion molecule-1 (PECAM-1/CD31) acts as a regulator of B-cell development, Bcell antigen receptor (BCR)-mediated activation, and autoimmune disease. Blood. 100:184-193

54. Becker KJ, McCarron RM, Ruetzler C, Laban O, Sternberg E, Flanders KC, Hallenbeck JM (1997) Immunologic tolerance to myelin basic protein decreases stroke size after transient focal cerebral ischemia. Proc Natl Acad Sci U S A 94:10873-10878
55. Reiss Y, Droste J, Heil M, Tribulova S, Schmidt MHH, Schaper W, Dumont DJ, Plate KH (2007) Angiopoietin-2 impairs revascularization after limb ischemia. Circ Res 101:88-96

56. Koo B-H, Coe DM, Dixon LJ, Somerville RPT, Nelson CM, Wang LW, Young ME, Lindner DJ et al (2010) ADAMTS9 is a cellautonomously acting, anti-angiogenic metalloprotease expressed by microvascular endothelial cells. Am J Pathol 176:1494-1504

57. Nishimori H, Shiratsuchi T, Urano T, Kimura Y, Kiyono K, Tatsumi $\mathrm{K}$ et al (1997) A novel brain-specific p53-target gene, BAI1, containing thrombospondin type 1 repeats inhibits experimental angiogenesis. Oncogene. 15:2145-2150

58. Gertz K, Kronenberg G, Kälin RE, Baldinger T, Werner C, Balkaya M, Eom GD, Hellmann-Regen J et al (2012) Essential role of interleukin-6 in post-stroke angiogenesis. Brain. 135:1964-1980

59. Hoffmann CJ, Harms U, Rex A, Szulzewsky F, Wolf SA, Grittner U et al (2015) Vascular signal transducer and activator of transcription-3 promotes angiogenesis and neuroplasticity longterm after stroke. Circulation. 131:1772-1782

60. Horie N, Pereira MP, Niizuma K, Sun G, Keren-Gill H, Encarnacion A, Shamloo M, Hamilton SA et al (2011) Transplanted stem cell-secreted vascular endothelial growth factor effects poststroke recovery, inflammation, and vascular repair. Stem Cells 29:274-285

61. Manoonkitiwongsa PS, Jackson-Friedman C, McMillan PJ, Schultz RL, Lyden PD (2001) Angiogenesis after stroke is correlated with increased numbers of macrophages: the clean-up hypothesis. J Cereb Blood Flow Metab 21:1223-1231

62. Gertz K, Kronenberg G, Uhlemann R, Prinz V, Marquina R, Corada $M$ et al (2016) Partial loss of VE-cadherin improves long-term outcome and cerebral blood flow after transient brain ischemia in mice. BMC Neurol 16:144

63. Gertz K, Priller J, Kronenberg G, Fink KB, Winter B, Schröck H et al (2006) Physical activity improves long-term stroke outcome via endothelial nitric oxide synthase-dependent augmentation of neovascularization and cerebral blood flow. Circ Res 99:1132-1140

64. Boon RA, Iekushi K, Lechner S, Seeger T, Fischer A, Heydt S, Kaluza D, Tréguer K et al (2013) MicroRNA-34a regulates cardiac ageing and function. Nature. 495:107-110

65. Yang Y, Cheng H-W, Qiu Y, Dupee D, Noonan M, Lin Y-D et al (2015) MicroRNA-34a plays a key role in cardiac repair and regeneration following myocardial infarction. Circ Res 117:450-459

66. Hattori Y, Okamoto Y, Maki T, Yamamoto Y, Oishi N, Yamahara K, Nagatsuka K, Takahashi R et al (2014) Silent information regulator 2 homolog 1 counters cerebral hypoperfusion injury by deacetylating endothelial nitric oxide synthase. Stroke. 45:34033411

67. Wan Y, Liu Y, Wang X, Wu J, Liu K, Zhou J et al (2015) Identification of differential microRNAs in cerebrospinal fluid and serum of patients with major depressive disorder. PLoS One 10:e121975

68. Bavamian S, Mellios N, Lalonde J, Fass DM, Wang J, Sheridan SD, Madison JM, Zhou F et al (2015) Dysregulation of miR-34a links neuronal development to genetic risk factors for bipolar disorder. Mol Psychiatry 20:573-584

69. Su W, Hopkins S, Nesser NK, Sopher B, Silvestroni A, Ammanuel $\mathrm{S}$ et al (2014) The p53 transcription factor modulates microglia behavior through microRNA-dependent regulation of c-Maf. J Immunol 192:358-366

Publisher's Note Springer Nature remains neutral with regard to jurisdictional claims in published maps and institutional affiliations. 